\title{
PERCEPÇÃO DE PUÉRPERAS SOBRE O TRABALHO DE PARTO CONDUZIDO POR ENFERMEIRAS OBSTÉTRICAS
}

\section{PERCEPTION OF PUERPERAL WOMEN ABOUT LABOR CONDUCTED BY OBSTETRIC NURSES}

\author{
Vallkrisnya Siqueira da Silva ${ }^{1} *$ Igho Leonardo do Nascimento Carvalho ${ }^{2} *$ Erisonval $^{*}$ \\ Saraiva da Silva ${ }^{3} *$ Luiza Eugênia Ferreira Mendes ${ }^{4} *$ Ingrid Moura de Abreu ${ }^{5}$ Ellen \\ Karem Rodrigues da Silva ${ }^{6}$
}

\begin{abstract}
RESUMO
Objetivo: Analisar a percepção das puérperas internadas no Centro de Parto Normal sobre a assistência prestada por enfermeiras obstétricas durante o trabalho de parto. Metodologia: Trata-se de um estudo qualitativo, descritivo, realizado por meio de entrevista com 15 puérperas internadas no Centro de Parto Normal. A coleta de dados aconteceu no período de janeiro a fevereiro de 2018, utilizando roteiro semiestruturado, contendo questões sobre caracterização sociodemográficas, sentimentos das puérperas e percepção sobre o acompanhamento das enfermeiras obstétricas durante o trabalho de parto. Resultados: As puérperas internadas no Centro de Parto Normal apresentaram sentimentos de satisfação apesar do medo, ansiedade e dor. A satisfação está relacionada ao atendimento recebido pela equipe de enfermagem, que tem o papel de aproximar a realidade da assistência à humanidade e as necessidades da mulher. Conclusão: A assistência dos profissionais de enfermagem pode auxiliar no enfrentamento de sentimentos e dúvidas, evidenciando um processo de acolhimento e satisfação principalmente pela forma de recepção e tratamento. Palavras-chaves: Parto Normal; Enfermeiras Obstétricas; Acolhimento.
\end{abstract}

\begin{abstract}
Objective: To analyze the perception of puerperal women admitted to the Centro de Parto Normal about the assistance provided by obstetric nurses during labor. Methodology: This is a qualitative, descriptive study, carried out through an interview with 15 puerperal women admitted to the Centro de Parto Normal. Data collection took place from January to February 2018, using a semi-structured script, containing questions about sociodemographic characterization, feelings of the puerperal women, perception of the monitoring of obstetric nurses during labor. Results: The puerperal women admitted to the Normal Childbirth Center showed feelings of satisfaction despite fear, anxiety and pain. This feeling is related to the care received by the nursing team, which has the role of approximating the reality of assistance to humanity and the needs of women. Conclusion: The assistance of nursing professionals can assist in coping with feelings and doubts, evidencing a process of reception and satisfaction, mainly due to the form of reception and treatment.
\end{abstract}

Keywords: Natural Childbirth; Nurse Midwives; User Embracement.

\footnotetext{
1 Enfermeira. Secretaria Municipal de Saúde de Arraial. Arraial, Piauí, Brasil. E-mail: vallkrisnya@hotmail.com ORCID: https://orcid.org/0000-0002-1220-6693

2 Enfermeiro. Doutor em Saúde Coletiva. Docente do Curso de Enfermagem da Universidade Federal do Piauí, Campus Amílcar Ferreira Sobral. Floriano, Piauí, Brasil. E-mail: ighocarvalho@gmail.com. ORCID: https://orcid.org/0000-0003-3165-9484

${ }^{3}$ Enfermeiro. Mestre em Saúde e Comunidade. Docente do Curso de Enfermagem da Universidade Federal do Piauí, Campus Amílcar Ferreira Sobral. Floriano, Piauí, Brasil. E-mail: erisonval@ gmail.com. ORCID: https://orcid.org/0000-0003-0286-9124

${ }^{4}$ Enfermeira. Especialista em obstetrícia. Coordenadora de Enfermagem do Serviço de Obstetrícia do Hospital Regional Tibério Nunes. Floriano, Piauí, Brasil. E-mail: enfalumendes@ gmail.com ORCID: https://orcid.org/0000-0002-5228-3757

${ }^{5}$ Enfermeira. Mestre e Doutoranda em Enfermagem. Docente do Curso de Enfermagem da Universidade Federal do Piauí, Campus Amílcar Ferreira Sobral. Floriano, Piauí, Brasil. E-mail: ingridmabreu@ outlook.com ORCID: https://orcid.org/000-0003-1785-606X ${ }^{6}$ Graduanda do Curso de Enfermagem da Universidade Federal do Piauí, Campus Amílcar Ferreira Sobral. Floriano, Piauí, Brasil. Email: hellen1973345@outlook.com ORCID: https://orcid.org/0000-0003-4248-7788
} 


\section{INTRODUÇÃO}

Os Centros de Parto Normal (CPN) são unidades de atendimento destinados a receber a parturiente e seu acompanhante para viabilizar um trabalho de parto ativo $\mathrm{e}$ participativo, diferenciando-se, assim, dos serviços tradicionais de atenção obstétrica ${ }^{(1)}$. Surgiu com o objetivo de resgatar o direito a privacidade e a dignidade da mulher ao dar a luz em um local semelhante ao seu ambiente familiar, oferecendo recursos tecnológicos apropriados em casos de eventual necessidade ${ }^{(2)}$.

A enfermeira obstétrica surge nesse cenário como profissional capaz de prestar uma assistência humanizada e de proporcionar às mulheres conforto $\mathrm{e}$ segurança ${ }^{(3)}$. Dessa forma, observa-se uma relação importante de vínculo entre as parturientes e as enfermeiras obstétricas, já que estas são responsáveis pela promoção do bem-estar no momento do parto ${ }^{(4)}$.

A humanização do parto é uma das ações que integram a Política Nacional de Humanização (PNH), desenvolvida pela Organização Mundial de Saúde (OMS), cuja premissa é o atendimento humanizado aos usuários do Sistema Único de Saúde (SUS), tendo o protagonismo na assistência ao parto exercido pelos profissionais de enfermagem obstétrica, o que reflete positivamente na redução de intervenções desnecessárias, como a prática excessiva de cesarianas ${ }^{(5)}$.

A valorização do parto humanizado aumenta a autonomia e estabelece uma relação dialógica entre o profissional de enfermagem obstétrica e a parturiente, contribuindo para uma boa evolução de trabalho de parto. O programa de humanização do parto tem seu início no prénatal, visto que a equipe de saúde prepara a parturiente para o momento do parto, promovendo o envolvimento como sujeita ativa, capaz e consciente do exercício dos seus direitos ${ }^{(6)}$.

Torna-se fundamental o incentivo ao parto normal, visto que promove diversos benefícios tanto para saúde da parturiente quanto do bebê. Nesse contexto, existe o reconhecimento da enfermagem obstétrica, como componente básico na assistência humanizada ao parto $^{(4)}$. Em 1993, foi criada a Rede de Humanização do Parto e Nascimento (ReHuNa) com o intuito de divulgar práticas humanizadas de assistência ao parto e nascimento, baseados em evidências científicas, e incentivar através do conhecimento que as mulheres tenham mais autonomia sobre seus corpos e $\operatorname{partos}^{(7)}$. Destarte, foram necessários movimentos como esses para que pudessem ser garantidos uma maior autonomia e direito das mulheres durante e depois do parto. 
Visto isso, o presente estudo objetiva analisar a percepção das puérperas internadas no Centro de Parto Normal sobre a assistência prestada por enfermeiras obstétricas durante o trabalho de parto. Dessa forma, adotou-se como questão norteadora: qual a percepção das puérperas sobre a assistência prestada pelas enfermeiras obstétricas durante $\mathrm{O}$ trabalho de parto?

\section{MÉTODO}

Trata-se de um estudo descritivo, com abordagem qualitativa, realizado no município de Floriano, Estado do Piauí, com 15 (quinze) puérperas submetidas ao trabalho de parto normal no CPN.

O CPN, local onde foi realizado o estudo, foi inaugurado em 07 de julho de 2017 e possui cinco quartos pré-parto, parto e pós-parto imediato (PPP) individuais, contando com uma equipe composta por 11 (onze) profissionais de enfermagem, sendo 5 (cinco) enfermeiras obstetras e 6 (seis) técnicos de enfermagem. O CPN ainda se encontra anexo ao hospital público regional, de médio porte, sendo que essa localização estratégica facilita o encaminhamento para o hospital, caso haja complicações durante o trabalho de parto.

Foram utilizados como critérios de inclusão: ser puérpera que tenha realizado o trabalho de parto normal no CPN, idade igual ou superior a 18 anos, estar em boas condições emocionais auto referidas. Os critérios de exclusão foram: não ter realizado acompanhamento pré-natal, possuir alguma doença de transmissão vertical auto referidas, tais como: HIV; sífilis; ocorrência do feto morto ou deformidade física, devido influenciarem sobre a percepção da experiência do trabalho de parto.

A coleta ocorreu no período de janeiro e fevereiro de 2018. As puérperas foram abordadas no CPN, no período de 06 - 18:00 horas após o parto normal, sendo convidadas a participar do estudo e esclarecidas quanto aos objetivos, riscos e benefícios de sua participação. $\quad$ Foram entrevistadas individualmente pela pesquisadora assistente, por um período de 20 a 30 minutos, evitando o horário da visita (16 - 17:00 h).

Optou-se pela utilização de um instrumento semiestruturado com questões sobre dados socioeconômicos e de condições de saúde, conhecimento, autonomia, percepção e sentimento das puérperas submetidas ao trabalho de parto normal conduzido por enfermeira obstétrica. Todas as entrevistas tiveram áudios gravados em aparelho de celular, tipo smartphone, com intuito de viabilizar a transcrição das respostas das questões subjetivas. Os dados socioeconômicos foram compostos por idade, localização, profissão/ocupação, religião, estado civil, escolaridade e renda familiar. Os dados das condições de saúde foram 
compostos por histórico gestacional, incluindo tipo de parto, abortamento, acompanhamento pré-natal, ocorrência de complicação e tempo de duração do trabalho de parto.

Os dados de conhecimento e autonomia das parturientes foram compostos por momento de admissão do parto, motivo da escolha do parto normal, escolha da posição de parir, direito a um acompanhante, percepção sobre a estrutura física do $\mathrm{CPN}$, se recebeu orientações sobre os procedimentos realizados, se o profissional explicou sobre os procedimentos realizados, sobre a experiência do parto normal realizado pela enfermeira obstétrica, o aspecto que mais a agradou durante o trabalho de parto normal, classificação da atenção do profissional de enfermagem e mensagem para outras gestantes.

As entrevistas foram identificadas em ordem numérica (Ex.: P1, P2, P3...) e realizadas até que se atingiu a saturação teórica dos dados. As questões objetivas foram agrupadas por meio de frequência absoluta, visando somente à caracterização das parturientes. Enquanto as questões subjetivas foram transcritas e analisadas conforme técnica de análise de conteúdo de Bardin, especificamente análise temática ${ }^{(8)}$.

Este estudo seguiu os preceitos éticos que regulamentam pesquisas que envolvem seres humanos, foi submetido e aprovado pelo
Comitê de Ética em Pesquisa com parecer $\mathrm{N}^{\circ}$ 3.661.818. Todas as participantes assinaram o Termo de Consentimento Livre e Esclarecido.

\section{RESULTADOS E DISCUSSÃO}

Foram entrevistadas um total de 15 (quinze) puérperas internadas no CPN. Pôdese evidenciar que a maioria reside nas cidades circunvizinhas a Floriano-PI (08), com faixa etária de 20 anos (10), se declara estudante (05), religião católica (13), casada/união estável (11). Quanto ao grau de escolaridade das puérperas, destaca-se a baixa escolaridade (1 ${ }^{\circ}$ Grau incompleto) (05), renda familiar menos de 1 salário mínimo (09). As participantes relataram histórico de gravidez anterior (10), sendo que a maioria foi de parto normal (09). Todas as puérperas realizaram consulta de pré-natal com enfermeiro (15), tendo como tempo de duração do parto em média de 4 (quatro) horas (04).

A realização do pré-natal com enfermeiros evidencia o incentivo ao parto normal na assistência de enfermagem realizada no acompanhamento pré-natal, bem como suas práticas de cuidado humanizado exercidos nesse processo $^{(9)}$ o que coincide com outro estudo que demonstra a importância do acompanhamento da gestação, afim de obter cada vez mais adesão ao prénatal, garantindo mais qualidade e melhor resultado obstétrico e perinatal ${ }^{(10)}$. 
Diante do estudo, as puérperas descreveram sentimentos positivos e negativos na admissão no CPN. Os sentimentos positivos foram a liberdade no trabalho de parto e o atendimento da equipe, conforme relatos abaixo:

Me trataram muito bem, porque eu estava falando até para minha mãe, porque ela já é a terceira e foi o melhor parto que tive, porque a enfermeira não ficou mandando fazer força, me deixou bem a vontade, eu fiquei caminhando no quarto, e quando fui para cama, já desceu(P5)

Tensa né, um pouco nervosa, mas ai a equipe do CPN me recebeu muito bem, me passou totalmente uma segurança me acalmou mais. Sei que a hora do parto é meio difícil, dor muita, mas em ver o choro do meu filho pela primeira vez foi emoção muito grande, até hoje feliz da vida por ele ter vindo ao mundo. Agradecer a equipe, enfermeiras obstétricas, psicólogos, todo mundo pelo atendimento. Excelente(P13)

Quando bem acompanhada e esclarecida, a experiência do parto pode se tornar um momento de reconhecimento da autonomia e da autoestima feminina, pois a enfermagem obstétrica tem papel fundamental na significação do parto e do nascimento para a mulher ${ }^{(11)}$. Dessa forma, OMS recomenda que a parturiente seja acompanhada por pessoas em quem confia e se sinta à vontade, especialmente por proporcionar confiança, segurança, apoio e força, capaz de reduzir a dor e a sensação de solidão, gerar bem-estar emocional e físico ${ }^{(12)}$.

Os sentimentos negativos do $\mathrm{CPN}$ foram nervosismo, dor, ansiedade e medo, conforme os relatos a seguir:

Me senti bem acolhida, nervosa, muito medo(P14)

Foi bom e ruim. A boa é ter o bebê, a ruim é a dor. Uma nota para a dor é 10, nunca tinha sentido antes $(P 4)$

Um pouco ansiosa, e também um pouco nervosa, mas graças a Deus estou bem (P6)

Alguns fatores podem aumentar a percepção da dor, tais como: medo, estresse, tensão, fadiga, frio, fome, solidão, desamparo social e afetivo, falta de conhecimento sobre os acontecimentos do parto e ambiente estranho $^{(11)}$. Então, deve-se atender às necessidades da mulher e do bebê, evitando intervenções desnecessárias e preservando a privacidade e autonomia das parturientes ${ }^{(13)}$.

Uma assistência obstétrica de qualidade necessita de cuidado, acolhimento, qualidade e resolutividade, evidenciando também a importância de um serviço de saúde que possua espaço para interações, com escuta sensível e ativa, com uma interação voltada para integralidade do cuidado, potencializando a sensibilidade humana, pois o parto é um vigoroso processo que envolve sensações físicas, emocionais e psíquicas, em que a mulher observa o 
ambiente, as pessoas e suas atitudes, além de estar voltada para si e para seu corpo ${ }^{(14)}$.

Além disso, as puérperas do $\mathrm{CPN}$ também apresentaram diferentes motivos para escolha do parto normal, tais como: recuperação e própria escolha, conforme relatos abaixo:

A dieta e a dor que também é só na hora, depois fica boa (P4)

Eu queria cesária para ligar, mas normal é muito melhor, porque depois pode sentar logo, ai eu achei até melhor (P5)

\section{Só não queria ter cesário (P10)}

Ainda houveram relatos de puérperas que não tiveram escolhas quanto ao parto normal, devido a decisão médica ou a condição clínica, conforme relatos abaixo:

Foi o médico que escolheu(P2)

Rapaz na verdade eu não tinha escolhido, ela se antecipou, mas não era para ser agora. Tive que ter normal, porque minha bebê estava sem líquido e estava laçada (P12)

A inserção das boas práticas no parto normal iniciou esse processo de desconstrução do modelo tecnológico, em prol da humanização da assistência, com base na classificação de condutas obstétricas no parto normal ${ }^{(15)}$. Logo, é necessário que essa conduta, promova cada vez mais espaço para discussão e desconstrução de mitos, já que, as mulheres são erroneamente influenciadas por estes, sobre dor exacerbada durante o parto natural, medo de mudanças com o corpo, comodidade e sobre a segurança de seu filho $^{(13)}$.

As puérperas do CPN também comentaram sobre a satisfação da estrutura física do local de parto humanizado, tais como: organização e admiração.

Ah, eu achei muito melhor do que a que tinha antigamente, em questão do apartamento, com questão da limpeza, a organização, da nova equipe que tem, super educada (P6)

A proposta da humanização inclui aspectos físicos, estruturais, recursos materiais, relações humanas, políticas organizacionais das instituições, aspectos culturais inerentes aos indivíduos, condições de trabalho, implementações das leis direcionadas ao público, capacidade do serviço de atender a demanda, entre outros ${ }^{(16)}$. Então, é possível concluir que a estrutura física de um CPN contribui de forma significativa para a satisfação das puérperas, haja vista que, faz parte do processo de humanização.

As puérperas do CPN ainda relataram seus sentimentos na experiência do parto normal realizado pela enfermeira obstétrica, tais como: satisfação pelo atendimento e rápida recuperação, e nervosismo, pois 
envolve dor, como observa-se nos depoimentos abaixo:

Melhor do que foi feito pelo médico, mais atenciosa, bem mais humanizado o parto, como eles chamam realmente $(P 8)$

Foi uma experiência assim digamos que um pouco nervosa, por ser uma enfermeira e não um médico profissional, fiquei assustada, ansiosa, mas graças a Deus deu tudo certo, apesar de ter ficado nervosa(P6)

Aconselho o parto humanizado, normal, é muito melhor, apesar da dor muito grande é, mas no momento que ver seu filho nascendo, chorando, você colocar no peito para mamar, não tem coisa melhor que isso, e também a recuperação é bem melhor, $e$ sempre manter a calma, não ficar tensa, porque tem profissionais capacitados para passar total segurança para o paciente (P13)

A humanização da assistência é importante para garantir que um momento único, como o parto, seja vivenciado de forma positiva e enriquecedora. Resgatar o contato humano, ouvir, acolher, explicar, criar vínculo são quesitos indispensáveis no cuidado $^{(17)}$. Então, é imprescindível que a parturiente esteja sempre disposta a participar de forma ativa no parto, o envolvimento efetivo da parturiente como sujeito ativo capaz de escolhas, contribui para que ela tenha consciência dos seus direitos e da sua autonomia $^{(13)}$.
Destarte, as puérperas do CPN relataram aspectos que mais as agradaram no parto normal conduzido por enfermeira obstétrica, focando no processo de trabalho humanizado, conforme os relatos:

A forma que eles estavam lidando comigo aqui, as orientações, medicação, tudo (P6)

A atenção dela, os métodos que ela usou, que vai ajudando, explicando, vai falando alguns exercícios para fazer que ajuda também, como a respiração(P8)

Nunca fui tratada assim em nenhum hospital (P5)

Logo, percebe-se a eficiência do uso de técnicas e métodos não farmacológicos, pois contribuem no alívio da dor, minimizam o nível de estresse e de ansiedade, promovendo satisfação, além de trazer benefícios que podem auxiliar na utilização de estratégias de cuidados que possam atender as necessidades específicas das parturientes ${ }^{(18)}$. Boas práticas obstétricas envolvem a comunicação entre os profissionais e a mulher ${ }^{(19)}$.

A humanização no processo de parir pressupõe atenção centrada na mulher, incentivando e encorajando durante todo processo, além disso, substituindo práticas intervencionistas por práticas menos invasivos e direcionando os cuidados a partir de condutas mais humanizadas(20-21). Nesse contexto, estudo $^{(22)}$ valida a ideia de que a 
assistência humanizada respeita $\mathrm{O}$ protagonismo da mulher, sua história, sua identidade e sua família.

Um fator relevante a ser citado é a representação que a parturiente sente ao ser acompanhada por pessoas em quem confia, pois se torna um alicerce de apoio emocional, e, uma vez que traz confiança e segurança, pode facilitar no processo de parturição. Diante disso, essa presença significa conforto, segurança e satisfação, produzidos pelo vínculo com o acompanhante e sentimento de respeito $^{(23)}$.

Para as puérperas, o bom atendimento resulta do interesse demonstrado no exame físico, perguntas feitas e orientações dadas, resolutividade das condutas adotadas e relações interpessoais ${ }^{(24)}$. No CPN, o processo de trabalho desenvolvido pelos profissinais da enfermagem obstétrica representa um diferencial, pois se baseia em relação dialógica num momento singular, no qual o respeito, os direitos e as necessidades de saúde são amplamente reconhecidas.

A atenção humanizada ao parto referese à necessidade de um novo olhar, compreendendo-o como uma experiência verdadeiramente humana. Acolher, ouvir, orientar e criar vínculo são aspectos fundamentais no cuidado às mulheres ${ }^{(25)}$.

Considerando a percepção das puérperas sobre o trabalho de parto normal conduzido por enfermeiras obstétricas, observa-se que o estudo promove contribuições a cerca do CPN, pois permite que sejam dsseminados os benefícios dessa estratégia de humanização ao parto, auxiliando também a enfermagem e os profissionais envolvidos a desenvolver ações humanizadas e de acordo com as políticas de saúde, pois a valorização do parto humanizado promove uma maior autonomia e influencia diretamente na vida da mulher.

O estudo apresenta como principal limitação o fato de que muitas puérperas optam por fazerem o parto normal em rede privada, provavelmente por insegurança de fazer o parto no CPN da rede pública e principalmente por ser um espaço comandado por enfermeiros, assim como não possuir cadastro na Atenção Primária à Saúde, o que é de certa forma um dado desproporcional, já que a maior parte das participantes da pesquisa são de baixa renda.

\section{CONCLUSÃO}

As puérperas que vivenciaram o parto no CPN apresentaram satisfação apesar do nervosismo e dor no momento do parto. Os fatores que contribuíram para essa satisfação foram: forma de recepção, tratamento, atenção e carinho recebido pela esquipe de enfermagem. Destarte, as participantes descreveram que a principal sensação no trabalho de parto é a dor. Entretanto, após o 
parto surge uma satisfação pelo alívio, contato com o filho, a rápida recuperação e ainda relataram surpresa com o conforto da estrutura física do CPN.

O estudo indicou a necessidade de matriciamento com os serviços de saúde da atenção básica, especialmente as equipes da Estratégia de Saúde da Família, o que iria potencializar o incentivo ao parto normal durante o acompanhamento pré-natal realizado por enfermeiros. Nesse contexto, as gestantes ainda seriam estimuladas a realizarem visita ao CPN para conhecerem estrutura física e atribuição do enfermeiro obstetra no trabalho de parto normal.

O trabalho de parto realizado pelo profissional de enfermagem obstétrica, no ambiente do CPN, representa uma experiência única para a mulher e seus familiares, devido a sua rápida recuperação e o fortalecimento do vínculo mãe e filho. Tal condição descreve a importância de especialização de profissionais de enfermagem e da estrutura na produção de saúde materna infantil, a partir do trabalho de parto seguro e humanizado.

É indispensável o acesso a informação para que as mulheres possam compreender sobre os benefícios do parto normal e a diminuição de riscos quando decidirem optar por ele, logo, é essencial ser estimulada a ser protagonista deste momento ímpar e sublime, favorecendo então uma parturição mais leve e prazerosa, haja vista que, o momento do parto marca efetivamente suas histórias.

Esta pesquisa contribuirá para ampliação do conhecimento sobre a compreensão das mulheres sobre o trabalho de parto humanizado realizado por profissionais da enfermagem obstétrica e cuidados prestados no CPN, ainda pode subsidiar a educação em saúde, estimulando a participação ativa das gestantes no trabalho de parto normal, instigando os profissionais a proporcionarem um ambiente acolhedor para estas parturientes. Espera-se que esse estudo ofereça subsídios da qualificação dos profissionais de enfermagem com o intuito de incentivar o parto normal realizado por enfermeiros obstetras.

\section{REFERÊNCIAS}

1. Garcia LV, Teles JM, Bonilha ALDL. O centro de parto normal e sua contribuição para atenção obstétrica e neonatal no Brasil. Revista Eletrônica Acervo Saúde [Internet]. 2017 [acesso em 22 mar 2021]; 7: S356-S363. Disponível em: https://www.lume.ufrgs.br/handle/10183/1705 57.

2. Pereira RM, Fonseca GO, Pereira ACCC, Gonçalves GA, Mafra RA. Novas práticas de atenção ao parto e os desafios para a humanização da assistência nas regiões sul e sudeste do Brasil. Ciência \& Saúde Coletiva [Internet]. 2018 [acesso em 18 fev 2021]; 23: 3517-3524. Disponível em: https://www.scielosp.org/article/csc/2018.v23 n11/3517-3524/. 
3. Alvares AS, Corrêa ACP, Nakagawa JTT, Teixeira RC, Nicolini AB, Medeiros RMK. Práticas humanizadas da enfermeira obstétrica: contribuições no bem-estar materno. Rev. Bras. Enferm. [Internet]. 2018 [acesso em 08 abrill 2021]; 71(Supp16):2620-2627. Disponível em:

http://www.scielo.br/scielo.php?script=sci_art text\&pid=S0034-

$71672018001202620 \& \operatorname{lng}=\mathrm{en}$.

4. Sousa AMM, Souza KVD, Rezende EM, Martins EF, Campos D, Lansky S. Práticas na assistência ao parto em maternidades com inserção de enfermeiras obstétricas, em Belo Horizonte, Minas Gerais. Escola Anna Nery [Internet]. 2016 [acesso em 22 mar 2021]; 20(2): 324-331. Disponível em: https://www.scielo.br/scielo.php?pid=S1414$81452016000200324 \&$ script=sci_arttext\&tlng $=$ pt.

5. Silva IA, Silva PSF, Andrade EWOF, Morais FF, Silva RSS, Oliveira LS. Percepção das puérperas acerca da assistência de enfermagem no parto humanizado. Revista Uningá [Internet]. 2017 [acesso em $14 \mathrm{fev}$ 2021]; 53(2): 37-43. Disponível em: http://revista.uninga.br/index.php/uninga/artic le/view/1440.

6. Sanches METL, Barros SMO, Santos AAP, Lucena TS. Obstetric nurse's role in the care of labor and chilbirth. Rev enferm UERJ [internet], Rio de Janeiro. 2019; 27:e43933. Doi:

http://dx.doi.org/10.12957/reuerj.2019.43933.

7. Fonseca I, Freitas RCM, Ril SY. Movimento de Doulas no Brasil: trajetória das atrizes na construção de uma cultura de não violência obstétrica. In: Seminário Internacional Fazendo Gênero11 \& Congresso mundos de mulheres. 2017 [acesso em 18 fev 2021]; p: 30-04. Disponível em:http://www.wwc2017.eventos.dype.com.b
r/resources/anais/1499436485_ARQUIVO_In araFonseca_TextoCompleto_MM_FG.pdf.

8. Minayo MCS. O desafio do conhecimento: pesquisa qualitativa em saúde. São Paulo: Hucitec/abrasco; 2008.

9. Alves TCM, Coelho ASF, Sousa MC, Cesar NF, Silva OS, Pacheco LR. Contribuições da enfermagem obstétrica para as boas práticas no trabalho de parto e parto vaginal. Enferm. foco [Internet]. 2019 [acesso em 14 fev 2021]; 10(4): 54-60. Disponível em:

https://pesquisa.bvsalud.org/portal/resource/pt /biblio-1052473.

10. Ribeiro JF, Luz VL, Sousa AS, Silva GLL, Feitosa VC, Almeida MF. Contribuição do pré-natal para o parto normal na concepção do enfermeiro da estratégia saúde da família. Revista Interdisciplinar [Internet]. 2016 [acrsso em 22 mar 2021]; 9(1): 161-170. Disponível em: http://revistainterdisciplinar.uninovafapi.edu.b r/index.php/revinter/article/view/521.

11. Freire HSS, Campos FC, Castro RCMB, Costa CC, Mesquita VJ, Viana RAA. Parto normal assistido por enfermeira: experiência e satisfação de puérperas. Rev. enferm. UFPE on line. 2017 [acesso em 14 fev 2021]; 11(6): 2357-2367. Disponível em: https://pesquisa.bvsalud.org/portal/resource/pt /bde-32163.

12. Brito VS, Guedes LFDSA, Prado LDOM, Menezes MO. Inserção do acompanhante no ciclo gravídico-puerperal: revisão integrativa da literatura. In Congresso Internacional de Enfermagem. 2017 [acesso em 22 mar 2021]; 1(1). Disponível em: https://eventos.set.edu.br/cie/article/viewFile/ $5620 / 2256$.

13. Pereira SS, Santos OICM, Silva SJB, Carvalho MCDMP. Parto natural: a atuação do enfermeiro diante da assistência humanizada. Tempus Actas de Saúde 
Coletiva [Internet]. 2016 [acesso em 22 mar 2021]; 10(3): ág-199. Disponível em: https://www.tempusactas.unb.br/index.php/te mpus/article/view/1727.

14. Gomes FES, Carvalho CE, Ferraz IC, Santos C.I, Barbosa GSL, Attem MS, et al. Impacto da implantação dos centros de parto normal na qualidade de assistência obstétrica. Research, Society and Development [Internet]. 2020 [acesso em 22 mar 2021]; 9(11): e3249119509e3249119509. Disponível em: https://www.rsdjournal.org/index.php/rsd/arti cle/view/9509.

15. Duarte MR, Alves VH, Rodrigues DP, Souza KV, Pereira AV, Pimentel MM. Tecnologias do cuidado na enfermagem obstétrica: contribuição para o parto e nascimento. Cogitare enferm [Internet]. 2019 [acesso em 14 fev 2021]; 24: e54164. Disponível em: https://core.ac.uk/download/pdf/328056200.p df .

16. Silva LNM, Silveira APKF, Morais FRR. Programa de humanização do parto e nascimento: aspectos instituicionais na qualidade da assistência. Rev. enferm. UFPE on line. 2017 [acesso em 14 fev 2021]; 11(supl.8): 3290-3294. Disponível em: https://pesquisa.bvsalud.org/portal/resource/pt /biblio-1032531.

17. Toral A, Vilain CRF, Morais $\mathrm{T}$, Valcarenghi V, Correia JBB, Ponciano TCL. Assistência de enfermagem na humanização do parto: uma revisão integrativa. Revista Eletrônica Estácio Saúde [Internet]. 2018 [acesso em 14 fev 2021]; 8(1): 45-53. Disponível em: http://periodicos.estacio.br/index.php/saudesa ntacatarina/article/view/5358.

18. Dias EG, Ferreira ARM, Martins AMC, Nunes MMJ, Alves JCS. Eficiência de métodos não farmacológicos para alívio da dor no trabalho de parto normal. Enferm. foco
[Internet]. 2018 [acesso em $14 \mathrm{fev} 2021$ ];9(2): 35-39. Disponível em: https://pesquisa.bvsalud.org/portal/resource/pt /biblio-1028353.

19. Monteiro BR, Souza NL, Silva PP, Pinto ESG, França DF, Andrade ACA, Oliveira AAV. Atenção à saúde no contexto do pré-natal e parto sob a perspectiva de puérperas. Revista Brasileira de Enfermagem [Internet]. 2020 [acesso em 14 fev 2021]; 73(4):e20190222. Disponível em: https://www.scielo.br/scielo.php?pid=S003471672020000400192\&script=sci_arttext\&tlng $=$ pt.

20. Melo R, Gomes GM, Fernandes FE, Pereira RC. Obstetric violence in the perception of professionals who attend. REAID [Internet]. 2020;91(29): 40-8. Doi: https://doi.org/10.31011/reaid-2020-v.91n.29-art.563.

21. Santos GG, Paço JAO. Visão e atuação humanizada de estudante do curso de Especialização em Enfermagem Obstétrica no parto normal. Global Academic Nursing Journal [Internet]. 2020 [acesso em 22 mar 2021]; 1(1): e9-e9. Disponível em: https://www.globalacademicnursing.com/inde x.php/globacadnurs/article/view/34.

22. Pinheiro GQ, Silva Júnior AP, Giotto AC. O processo do parto: a importância do enfermeiro no parto humanizado. REIcEN [Internet]. 2019 [acesso em 4 abr 2021]; 2(4): 190-5. Disponível em: https://revistasfacesa.senaaires.com.br/index.p hp/iniciacao-cientifica/article/view/255.

23. Ferreira KA, Madeira LM. The meaning of the companion in the childbirth for woman and relatives. Enfermagem Obstétrica [Internet]. 2016 [acesso em 22 mar 2021]; 3: e29. Disponível em: http://www.enfo.com.br/ojs/index.php/EnfOb st/article/view/29. 
24. Corrêa MSM, Feliciano KVO, Pedrosa EN, Souza AI. Acolhimento no cuidado à saúde da mulher no puerpério. Cadernos de saúde pública [Internet]. 2017 [acesso em 14 fev 2021]; 33: e00136215. Disponível em: https://www.scielosp.org/article/csp/2017.v33 n3/e00136215/.

25. Possati AB, Prates LA, Cremonese L, Scarton J, Alves CN, Ressel LB. Humanização do parto: significados e percepções de enfermeiras. Escola Anna Nery Revista de Enfermagem [Internet]. 2017 [acesso em 14 fev 2021]; 21(4): 1-6. Disponível em: https://www.redalyc.org/pdf/1277/127752022 003.pdf.

Submissão: $2021-04-15$

Aprovado: 2021-04-25 